\title{
Mediastinal Lymphoma Presenting in Cardiogenic Shock with Superior Vena Cava Syndrome in a Primigravida at Full Term: Salvage Resection after Prolonged Extracorporeal Life Support
}

\author{
Sabrina E. Carro ${ }^{a}$ David W. Essex ${ }^{b}$ Mohamed Alsammak $^{c}$ \\ Ashish Bains $^{c}$ Yoshiya Toyodad ${ }^{d}$ Suresh Keshavamurthy ${ }^{d}$ \\ aMD Candidate 2020, Lewis Katz School of Medicine at Temple University, \\ Philadelphia, PA, USA; ${ }^{b}$ Department of Hematology and Oncology, Temple University \\ Hospital, Philadelphia, PA, USA; 'Department of Pathology and Laboratory Medicine, \\ Temple University Hospital, Philadelphia, PA, USA; ${ }^{d}$ Department of Cardiovascular \\ Surgery, Temple University Hospital, Philadelphia, PA, USA
}

\section{Keywords}

PMBCL · Non-Hodgkin - Lymphoma, mediastinal mass - Salvage resection · Extracorporeal membrane oxygenation · ECMO $\cdot$ Pathology $\cdot$ Surgery

\section{Abstract}

Primary mediastinal large $\mathrm{B}$-cell lymphoma (PMBCL) is a rare type of non-Hodgkin lymphoma that typically has a good response rate to first line chemotherapy regimens. There have been reports of successful chemotherapy, but with a residual mass from fibrosis. Here, we report the case of a 19-year-old primigravida presenting with cardiogenic shock and superior vena cava 
(SVC) syndrome at full term who was found to have a PMBCL. Following delivery via urgent cesarean section, she was put on veno-arterial extra corporeal membrane oxygenation (VAECMO) and once hemodynamically stable was started on chemotherapy. In view of limited change in tumor size on consecutive CT scans and questionable response to chemotherapy, there were multidisciplinary meetings wherein withdrawing support was discussed and put forward to the family. At that point, surgical debulking was offered on compassionate grounds to be able to wean her off the VA-ECMO. This case report highlights the role of salvage resection when there are no other options.

(c) 2019 The Author(s)

Published by S. Karger AG, Basel

\section{Introduction}

Primary mediastinal large B-cell lymphoma (PMBCL) is a rare type of non-Hodgkin lymphoma often seen in reproductive females, comprising approximately $2-3 \%$ of all non-Hodgkin lymphomas $[1,2]$. Lymphomas are the fourth highest cancer seen in pregnancy with Hodgkin lymphoma as the most common followed by non-Hodgkin lymphoma affecting about 5.4 per 1,000 pregnancies [2]. PMBCL arises from the thymus, usually invading local structures presenting as cough, dyspnea, chest pain, superior vena cava (SVC) syndrome, pleural or pericardial effusion while B symptoms, such as fever, night sweats, and weight loss are seen in less than $20 \%$ of patients with PMBCL $[1,3,4]$. Most PMBCL respond well to chemotherapy; however, if refractory, treatment options include salvage chemotherapy with or without radiation. There are also cases of residual mediastinal mass reported after successful therapy [5]. A multidisciplinary approach to PMBCL is common; however, complete management strategies are underreported. Here we report a case of a PMBCL in a young woman and the importance of multidisciplinary management, specifically the use of surgical resection in her complicated course.

\section{Case Report/Case Presentation}

A 19-year-old primigravida with a history of asthma and psoriasis presented at 39 weeks gestation to a local hospital with increasing shortness of breath. She was found to have a white out of the right chest in addition to large pericardial effusion. The effusion was drained in an attempt to stabilize her; however, she developed signs and symptoms of cardiogenic shock including tachycardia, tachypnea, and hypotension resulting in an emergent bedside caesarian section, giving birth to a healthy baby, during which she required cardioversion for supraventricular tachycardia. Postpartum chest CT demonstrated an extremely large anterior mediastinal mass measured at $14.0 \times 10.6 \times 15.6 \mathrm{~cm}$, which was determined by flow cytometry on fine needle aspiration specimen to comprise a predominant population of clonal kapparestricted B cells. Core biopsy showed a B-cell lymphoma with positive immunohistochemical staining for CD20, PAX5, BCL6, and occasional cells expression of CD30 and CD23, while CD10 expression was weak to equivocal and BCL2 and MUM1 were negative. Proliferation index is estimated at $70 \%$ by Ki-67. FISH analysis is negative for BCL2, BCL6, and MYC gene rearrangement excluding high grade B-cell lymphoma. The tissue also showed extensive necrosis. The 
Carro et al.: Mediastinal Lymphoma Presenting in Cardiogenic Shock with Superior Vena Cava Syndrome in a Primigravida at Full Term

final diagnosis given was a primary mediastinal large B-cell lymphoma (PMBCL) in the absence of evidence of systemic disease by radiologic studies.

Serial echocardiograms demonstrated worsening ejection fractions (EF) of 55-60\% to $10 \%$ causing her to be transferred to our institution for hemodynamic support. After arrival, she became hypoxemic and hypotensive, requiring initiation of advanced circulatory support. This resulted in the placement of veno-arterial extra corporeal membrane oxygenation (VAECMO) percutaneously employing $17 \mathrm{~F}$ left proximal femoral arterial, $7 \mathrm{~F}$ left distal femoral arterial, and $25 \mathrm{~F}$ left femoral venous cannulae. Over the next two months, she continued to have a complicated course consisting of postpartum cardiomyopathy, large right middle cerebral artery stroke, ocular compartment syndrome, urinary tract infections, multidrug resistant ventilator associated pneumonia, deep vein thromboses, left ventricle thrombus, superior vena cava syndrome, and bilateral mainstem bronchial compression (Table 1). Chemotherapy was initiated on Hospital Day 3 consisting of R-CEOP (Table 2) with etoposide replacing doxorubicin in the setting of heart failure. R-CHOP (Table 2) was used in cycle 2, Hospital Day 24, as the EF had improved to 60\%. The patient remained on VA-ECMO for 54 days, an unusually long time, as support throughout her treatment.

The second CT scan (Fig. 1), on Hospital Day 40, indicated minimal change in tumor size of $13.2 \times 8.2 \times 13.2 \mathrm{~cm}$ with worsening lung collapse bilaterally and little lung aeration. A tumor board meeting was held with multiple physicians from thoracic medicine and surgery, hematology and oncology, nuclear medicine, interventional cardiology, and pathology to discuss the next step taking into consideration worsening thrombocytopenia, risks and benefits of continuing dependence on VA-ECMO, and worsening mass effect on the heart, bilaterally main stem bronchi, and superior vena cava. The poor clinical response was suspected to be from fibrosis and necrosis with edema of the tumor rather than a primary refractory PMBCL. Due to the patient's fragile hemodynamic state, salvage chemotherapy was initially considered rather than a debulking surgery; however, ultimately, after the tumor board meeting, an ethics board meeting, and a family meeting, the decision was made to withdraw further treatment. At that juncture, on compassionate grounds, a salvage resection was offered by Cardiovascular surgery.

The patient underwent a median sternotomy to access the tumor. There were extensive collateral vessels due to the SVC syndrome. The tumor extended from the right upper lobe to the pericardium compressing the SVC (Fig. 2A). There was no evidence of pericardial or heart infiltration and the aorta and arch vessels seemed intact externally. There was moderate hemopericardium and adhesions to the right lung. Two large pieces $(8.5 \times 7.5 \times 4.5 \mathrm{~cm}$ and 15 $\times 12.5 \times 6.5 \mathrm{~cm}$ ) were removed (Fig. 2C) with pathology showing focally preserved areas of CD20+ large B-cells consistent with the patient's initial diagnosis as well as areas of fibrosis and extensive necrosis (Fig. 3A-D). An unresected portion of the tumor remained adjacent to the right upper lobe (Fig. 2B). At this time, TEE demonstrated an EF of 50-55\% and bronchoscopy revealed bilateral patent bronchi. The chest cavity was left open due to coagulopathy. Throughout surgery, the patient received multiple blood products and red blood cells. The surgical site was lavaged with distilled water because the hypotonic properties assist in tumor cell lysis. Two days later, she returned to the operating room for a successful ECMO decannulation. Her chest was closed with a sternal plate and wires for reinforcement. Bronchoscopy at this time revealed mucus plugging of the right upper lobe. 


\section{Case Reports in Oncology}

Case Rep Oncol 2019;12:401-410

DOI: $10.1159 / 000499195$

2019 The Author(s). Published by S. Karger AG, Base www.karger.com/cro

Carro et al.: Mediastinal Lymphoma Presenting in Cardiogenic Shock with Superior Vena Cava Syndrome in a Primigravida at Full Term

A few days prior to the partial resection, the distal perfusion cannula to the left femoral artery was dislodged and attempts to replace it were unsuccessful. Hemostasis was accomplished by compression. The arterial cannula was then placed in the right femoral artery. The left leg showed signs of progressively worsening ischemia requiring vascular surgery to be consulted. Fasciotomy was performed and a shunt was placed from the aorta to the left superficial femoral artery due to an occlusion from the external iliac to the superficial femoral artery. The shunt, however, clotted and her left foot became cold and mottled requiring a through knee guillotine amputation. This approach was performed as a life saving approach due to her continuing comorbidities rather than attempting to save the limb and put the patient at more unnecessary risk.

\section{Discussion/Conclusion}

Primary mediastinal large B-cell lymphoma (PMBCL) is a rare type of non-Hodgkin lymphoma comprising approximately $2-3 \%$ of all non-Hodgkin lymphomas $[1,2]$. Although historically viewed akin to diffuse large B-cell lymphoma, gene expression of PMBCL is more similar to nodular sclerosis Hodgkin lymphoma $[1,6]$. PMBCL tumor cells are positive for B-cell markers, and potentially a variety of nuclear transcriptional regulators amongst other gene expression alterations [4]. R-CHOP and dose adjusted EPOCH-R are the primary chemotherapy regimens to treat PMBCL because of their high response and cure rates $[4,5]$. If there is minimal change on reimaging after the designated cycles of chemotherapy, there is high suspicion for either a primary refractory lymphoma or a residual tumor consisting of fibrosis and necrosis $[5,7]$. A primary refractory lymphoma would warrant salvage chemotherapy consisting of ICE or R-ICE while if pathology is determined to be fibrosis and necrosis, the patients would be eligible for surgical resection having a more favorable prognosis with complete resection [8].

There is minimal literature discussing anterior mediastinal mass resection due to its rare occurrence. Bacha et al. [8] discuss treatment involving surgical resection and postoperative outcomes. The 5 -year survival rate for chemotherapy followed by surgery in lymphomas was $83 \%$, whereby the 5-year survival rate for subtotal resection of this type of mass was just below 30\% [8]. Since this time, technological advances have allowed safer perioperative management of these patients [9]. This includes preoperative ECMO cannulation (as seen in our patient) or cardiopulmonary bypass cannulation in the event of a cardiovascular collapse to prevent neurologic injury, as well as general advances in imaging modalities to better guide surgical techniques and challenges that face the surgical and anesthetic team [9-11]. However, ECMO does not come without its complications. Our patient spent 54 days on VA-ECMO, increasing the likelihood of complications such as hemorrhage, hyperperfusion, limb ischemia, upper body hypoxemia, compartment syndrome, fasciotomy, amputation, stroke, hypoxic brain injury, and mortality [12]. In a study of cannulation-related complication of VAECMO by Wong et al. [12], 11\% experienced cannula related hemorrhage, $25 \%$ experienced limb ischemia, and 53\% had a 30-day mortality in those with femoral ECMO cannulation.

Here we report the successful management of a complicated case of a giant PMBCL with VA-ECMO and debulking of the residual tumor. For our patient's cardiopulmonary collapse caused by tumor compression, we utilized VA-ECMO to maintain her life. Although these 
tumors typically respond well to first line chemotherapies, our patient's tumor's lack of response on CT imaging necessitated the reevaluation of surgery. Despite her extensive comorbidities, we proceeded with surgical debulking to relieve the compression in order to wean VA-ECMO. This case highlights the importance of surgical resection consideration in the management of these rare mediastinal masses.

\section{Statement of Ethics}

Informed consent was gained from the relative for publication of this case report and the accompanying images.

\section{Disclosure Statement}

The authors have no conflicts of interest to declare.

\section{Funding Sources}

Publication of this article was funded in part by the Temple University Libraries Open Access Publishing Fund.

\section{Author Contributions}

All authors contributed to the content of this manuscript.

\section{References}

1 Martelli M, Ferreri A, Di Rocco A, Ansuinelli M, Johnson PW. Primary mediastinal large B-cell lymphoma. Crit Rev Oncol Hematol. 2017 May;113(6):318-27.

2 Fiascone S, Datkhaeva I, Winer ES, Rizack T. Primary mediastinal large B-cell lymphoma in pregnancy. Leuk Lymphoma. 2016;57(1):240-3.

3 Yamamoto W, Nakamura N, Tomita N, Ishii Y, Takasaki H, Hashimoto C, et al. Clinicopathological analysis of mediastinal large B-cell lymphoma and classical Hodgkin lymphoma of the mediastinum. Leuk Lymphoma. 2013 May;54(5):967-72.

4 Bhatt VR, Mourya R, Shrestha R, Armitage JO. Primary mediastinal large B-cell lymphoma. Cancer Treat Rev. 2015 Jun;41(6):476-85.

5 Dunleavy K. Primary mediastinal B-cell lymphoma: biology and evolving therapeutic strategies. Hematology Am Soc Hematol Educ Program. 2017 Dec;2017(1):298-303.

6 Vardhana S, Hamlin PA, Yang J, Zelenetz A, Sauter CS, Matasar MJ, et al. Outcomes of Relapsed and Refractory Primary Mediastinal (Thymic) Large B Cell Lymphoma Treated with Second-Line Therapy and Intent to Transplant. Biol Blood Marrow Transplant. 2018 Oct;24(10):2133-8.

7 Lazarovici J, Terroir M, Arfi-Rouche J, Michot JM, Mussot S, Florea V, et al. Poor predictive value of positive interim FDG-PET/CT in primary mediastinal large B-cell lymphoma. Eur J Nucl Med Mol Imaging. 2017 Nov;44(12):2018-24. 
Carro et al.: Mediastinal Lymphoma Presenting in Cardiogenic Shock with Superior Vena Cava Syndrome in a Primigravida at Full Term

8 Bacha EA, Chapelier AR, Macchiarini P, Fadel E, Dartevelle PG. Surgery for invasive primary mediastinal tumors. Ann Thorac Surg. 1998 Jul;66(1):234-9.

9 Slinger P, Karsli C. Management of the patient with a large anterior mediastinal mass: recurring myths. Curr Opin Anaesthesiol. 2007 Feb;20(1):1-3.

10 Li WW, van Boven WJ, Annema JT, Eberl S, Klomp HM, de Mol BA. Management of large mediastinal masses: surgical and anesthesiological considerations. J Thorac Dis. 2016 Mar;8(3):E175-84.

11 Tempe DK, Arya R, Dubey S, Khanna S, Tomar AS, Grover V, et al. Mediastinal mass resection: femorofemoral cardiopulmonary bypass before induction of anesthesia in the management of airway obstruction. J Cardiothorac Vasc Anesth. 2001 Apr;15(2):233-6.

12 Wong JK, Melvin AL, Joshi DJ, Lee CY, Archibald WJ, Angona RE, et al. Cannulation-Related Complications on Veno-Arterial Extracorporeal Membrane Oxygenation: Prevalence and Effect on Mortality. Artif Organs. 2017 Sep;41(9):827-34.

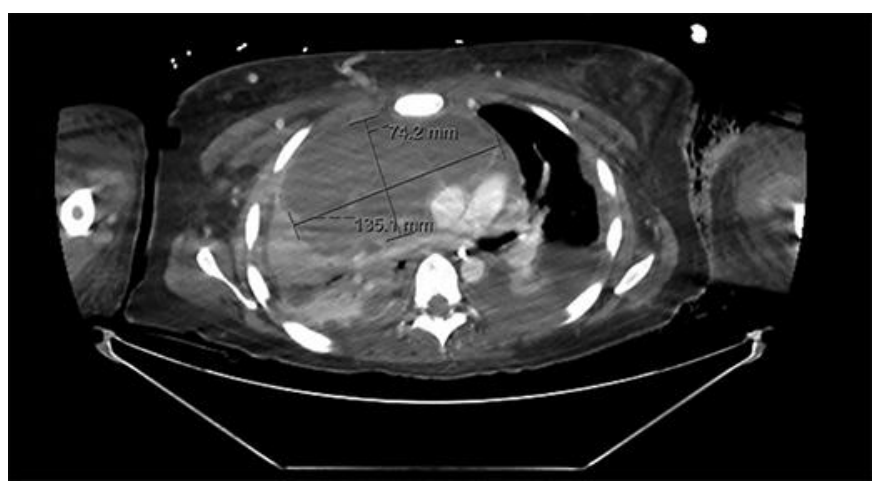

Fig. 1. Chest CT with contrast on Hospital Day 40 after two cycles of chemotherapy. 


\section{Case Reports in Oncology}



Fig. 2. A. Tumor (blue arrow) being resected in two pieces via electrocautery. B. View of the chest posttumor debulking. The pericardium can be seen held up by sutures with the heart to the left with a portion of unresected tumor (blue arrow) proximal of the pericardium. The base of the right lung can be visualized on the right side of the open chest cavity. C. One piece of the resected tumor. 


\section{Case Reports in Oncology}

Case Rep Oncol 2019;12:401-410

DOI: $10.1159 / 000499195$

(c) 2019 The Author(s). Published by S. Karger AG, Basel www.karger.com/cro

Carro et al.: Mediastinal Lymphoma Presenting in Cardiogenic Shock with Superior Vena Cava Syndrome in a Primigravida at Full Term

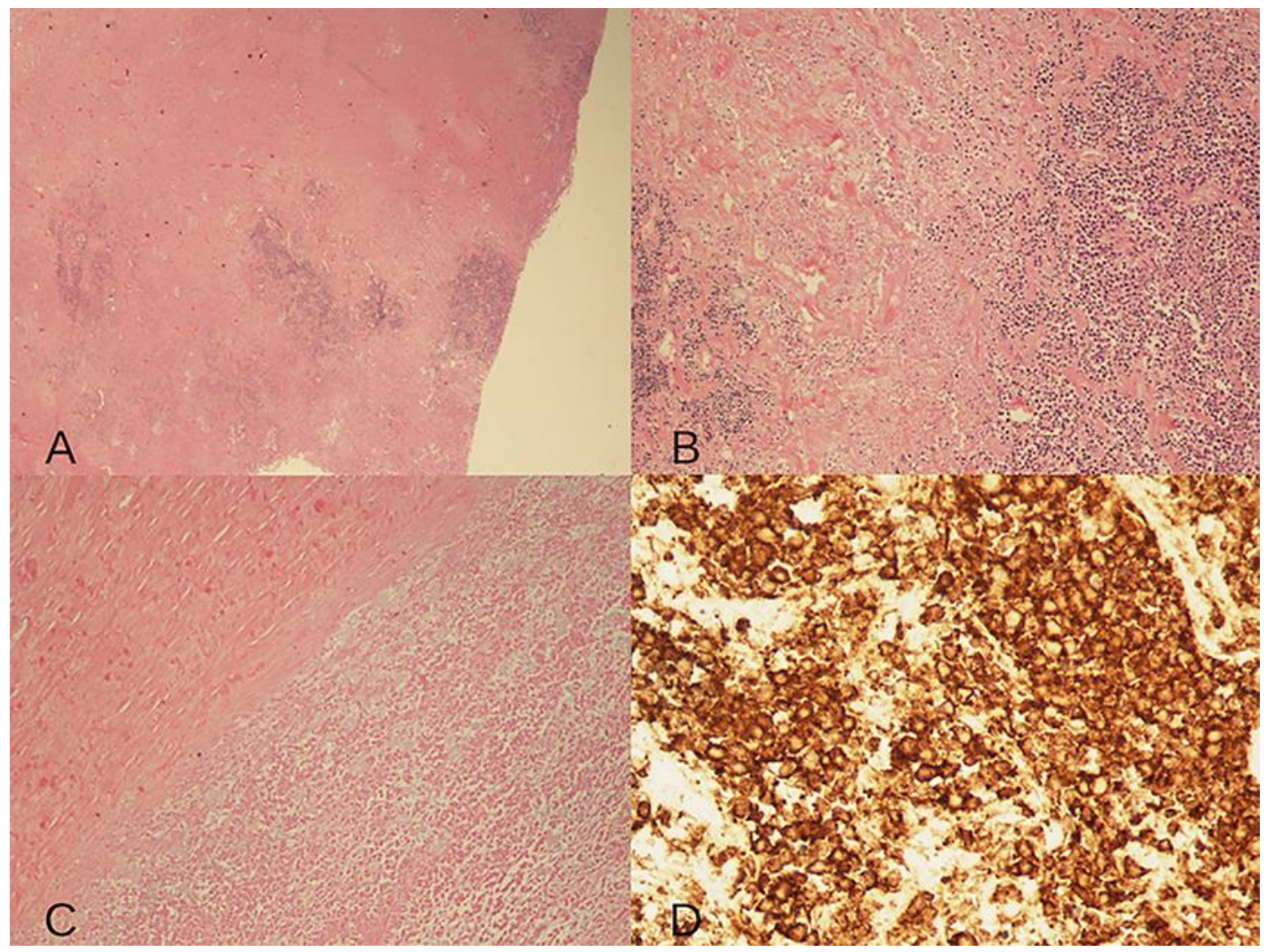

Fig. 3. A-C. Sections of the resected specimen demonstrating focally preserved lymphoma surrounded by extensive fibrosis and necrosis (H\&E stain). D. Preserved lymphoma cells positive for CD20. 
Table 1. Hospital course overview

\author{
Hospital Course Events \\ (Day) \\ Pulseless electrical activity arrest, VA-ECMO cannulation; Pathology report from outside \\ hospital indicates B-cell lymphoma with necrotic features \\ $2 \quad$ Large right middle cerebral artery stroke \\ 3 Rituximab of R-CEOP started. Bilateral upper extremity DVTs. EF 5-10\%; LV thrombus \\ visualized \\ $4 \quad$ CEOP portion of R-CEOP begun \\ $7 \quad$ Ocular compartment syndrome requiring bilateral canthotomy \\ 11 EF 45-50\%; LV thrombus still present \\ 17 Tracheostomy \\ 19 EF 55-60\%; LV thrombus unchanged \\ 24 CT Chest; R-CHOP begun \\ 27 EF 65-70\%; LV thrombus present \\ 33 Provera started due to continued vaginal bleeding \\ 34 Bilateral upper extremity DVTs still present; EF 20-25\% \\ 38 EF 25-30\%; LV thrombus on repeat echo. \\ $40 \quad$ Repeat CT Chest \\ $41 \quad$ Tumor Board meeting \\ 43 Reperfusion cannula dislodged, Massive transfusion protocol activated \\ 46 DIC \\ 48 Moderate pericardial effusion; EF 65-70\%; LV Thrombus present \\ $49 \quad$ Ethics Meeting \\ $52 \quad$ Debulking surgery; EF $65-70 \%$ \\ 54 Weaned off VA-ECMO; EF $50 \%$ \\ $56 \quad$ Guillotine amputation at left knee \\ $61 \quad$ EF $60 \%$ \\ 98 Sternal debridement, bilateral pectoris major muscle flap, hardware removal \\ $136 \quad$ Ultrasound showing diaphragmatic dysfunction \\ 144 At the time of this submission, the patient is still in our care with the goal of being \\ discharged to a long term acute care facility in the near future
}

Note: this just highlights some of the events our patient experienced. In addition to what is noted, she also had many blood product transfusions, experienced numerous infections with multidrug resistant organisms, and required multiple interventions to assist with cardiovascular function. 


\section{Case Reports in Oncology}

\begin{tabular}{l|l}
\hline Case Rep Oncol 2019;12:401-410 \\
\hline DOI: 10.1159/000499195 & $\begin{array}{l}\text { (c) 2019 The Author(s). Published by S. Karger AG, Basel } \\
\text { www.karger.com/cro }\end{array}$
\end{tabular}

Carro et al.: Mediastinal Lymphoma Presenting in Cardiogenic Shock with Superior Vena Cava Syndrome in a Primigravida at Full Term

Table 2. Chemotherapy acronyms

\section{Chemotherapy acronyms Description}

$\mathrm{R}$

CHOP

EPOCH

ICE
Rituximab

Cyclophosphamide, doxorubicin, vincristine, prednisone

Etoposide, prednisone, vincristine, cyclophosphamide, doxorubicin Isofamide, carboplatin, etoposide 